\title{
Blocking Interleukin-4 Receptor $\alpha$ Using Polyethylene Glycol Functionalized Superparamagnetic Iron Oxide Nanocarriers to Inhibit Breast Cancer Cell Proliferation
}

\author{
Abjal Pasha Shaik, $\mathrm{PhD}^{1}$ \\ Asma Sultana Shaik, $\mathrm{PhD}^{2}$ \\ Ali Al Majwal, $\mathrm{PhD}^{3}$ \\ Achraf Al Faraj, $\mathrm{PhD}^{4}$
}

\begin{abstract}
Purpose
The specific targeting of interleukin-4 receptor $\alpha(I L 4 R \alpha)$ receptor offers a promising therapeutic approach for inhibition of tumor cell progression in breast cancer patients. In the current study, the in vitro efficacy of superparamagnetic iron oxide nanoparticles conjugated with anti-IL4R $\alpha$ blocking antibodies (SPION-IL4R $\alpha$ ) via polyethylene glycol polymers was evaluated in 4T1 breast cancer cells.
\end{abstract}

\section{Materials and Methods}

Cell viability, reactive oxygen species generation, and apoptosis frequency were assessed in vitro in 4T1 cancer cell lines following exposure to SPION-IL4R $\alpha$ alone or combined with doxorubicin. In addition, immunofluorescence assessments and fluorimetry were performed to confirm the specific targeting and interaction of the developed nanocarriers with IL4R $\alpha$ receptors in breast cancer cells.

\section{${ }^{1}$ Department of Clinical Laboratory Sciences, College of Applied Medical Sciences,}

King Saud University, Riyadh,

${ }^{2}$ Prince Naif Health Research Center, College of Medicine, King Saud University, Riyadh, Departments of ${ }^{3}$ Community Health Sciences and ${ }^{4}$ Radiological Sciences, College of Applied Medical Sciences, King Saud University, Riyadh, Saudi Arabia

\section{Results}

Blocking of IL4R $\alpha$ receptors caused a significant decrease in cell viability and induced apoptosis in $4 \mathrm{~T} 1$ cells. In addition, combined treatment with SPION-IL4R $\alpha+$ doxorubicin caused significant increases in cell death, apoptosis, and oxidative stress compared to either SPIONIL4R $\alpha$ or doxorubicin alone, indicating the enhanced therapeutic efficacy of this combination. The decrease in fluorescence intensity upon immunofluorescence and fluorimetry assays combined with increased viability and decreased apoptosis following the blocking of IL4R $\alpha$ receptors confirmed the successful binding of the synthesized nanocarriers to the target sites on murine $4 \mathrm{~T} 1$ breast cancerous cells.

\section{Conclusion}

These results suggest that SPION-IL4R $\alpha$ nanocarriers might be used for successful reduction of tumor growth and inhibition of progression of metastasis in vivo.
Department of Radiological Sciences,

College of Applied Medical Sciences,

King Saud University, Riyadh 11433,

Saudi Arabia

Tel: 966-11-46-99664

Fax: 966-11-46-93565

E-mail: achraf.alfaraj@gmail.com

Received February 29, 2016

Accepted June 16, 2016

Published Online July 12, 2016

\section{Key words}

Breast neoplasms, Dextran coated superparamagnetic iron oxide, Biomarkers, Tumor, Receptors, Interleukin-4, Drug delivery systems

\section{Introduction}

Breast cancer remains the most common malignant tumor in women worldwide and one of the principal causes of cancer deaths [1]. Breast cancer cell growth is modulated by a composite network of growth factors, chemokines, and cytokines that induce rapid cell proliferation and metastasis [2]. Cytokines are important signaling molecules in the tumor microenvironment that regulate cancer cell growth through cancer cell progression and metastasis. Murine breast cancer models revealed that interleukin (IL) 4 and IL-13 secretion and activation of IL-4 receptor signaling facilitated tumor cell metastasis [3]. Both IL-4 and IL-13 are dependent on the interleukin- 4 receptor $\alpha(\operatorname{IL} 4 R \alpha)$ chain either for recognition and signaling (i.e., IL-4) or signal transduction (i.e., IL-13). IL-4 binds to the type I receptor, whereas both IL-4 and IL-13 bind to the IL4R $\alpha$ type II receptor. In fact, 
an upregulation in the expression of IL4R $\alpha$ has not only been seen in breast cancer cells, but also in rhabdomyosarcoma [4], bladder cancer [5], and brain cancer [6]. Owing to the role of this receptor in regulation of cell division, it can be interpolated that the IL4/IL4R $\alpha$ interaction and consequent signaling changes within cells can promote not only the proliferation, but also the survival of breast cancer cells. Therefore, antibodies that specifically block IL4R $\alpha$ can prevent cancer cell proliferation and metastasis.

Targeted delivery to specific cells through the use of nanoscale drug delivery systems (DDSs) allows enhanced and selective accumulation of therapeutic agents in disease areas [7]. In addition, nanocarriers can protect the drug/ inhibitor against degradation and increase its half-life in the bloodstream, allowing better bio-pharmacokinetic control in host tissues [8]. Therefore, enhancing the ability of blocking antibodies to be administered directly to the cancerous tissue may improve treatment efficacy and decrease systemic side effects.

Recent advances in nanoscale DDS allow specific delivery of various treatment options to the target tissue, ensuring improved efficacy, decreased side effects, and controlled release. Among the different nanoparticles used, superparamagnetic iron oxide nanoparticles (SPION) offers a novel theranostic approach enabling high detection sensitivity and selective targeting. Biomedical interest in SPIONs has increased considerably in the last few decades because of their potential for use as contrast agents in magnetic resonance imaging (MRI), in magnetic separation techniques, and hyperthermia. Furthermore, SPIONs have superior biocompatibility and ability to cross biological membranes in addition to providing the possibility of achieving high drugloading capacity, sustained release, enhanced drug stability and absorption, as well as targeted deposition $[9,10]$. Doxorubicin (DOX), a member of the anthracycline class of chemotherapeutic agents, is commonly used to treat several common human cancers [11]. Therefore, targeting IL4R $\alpha$ using SPION conjugated with anti-IL4R $\alpha$ blocking antibodies (SPION-IL4R $\alpha$ ) nanoparticles in combination with low doses of DOX may help reduce toxicity and improve the clinical usefulness of cancer therapy.

This study was conducted to assess the in vitro efficacy of IL4R $\alpha$ conjugated SPION as theranostic nanoprobes for breast cancer by evaluating cell viability, reactive oxygen species generation, and apoptosis frequency in 4T1 breast cancer cells. In addition, the successful interaction and targeting of anti-IL4R $\alpha$ antibody conjugated SPION with IL4R $\alpha$ receptors on 4T1 cancer cells was confirmed and the efficacy of combined treatment with SPION-IL4R $\alpha$ and DOX was reported.

\section{Materials and Methods}

\section{SPION-IL4R $\alpha$ design and characterization}

Nanoferrite SPIONs prepared via the core-shell method with a core of $75 \%-80 \%(\mathrm{w} / \mathrm{w})$ magnetite and a shell of dextran (Micromod Partikeltechnologie $\mathrm{GmbH}$, Rostock, Germany) were used in this study. SPIONs were conjugated to polyethylene glycol (PEG) polymer with a molecular weight of 2,000 g/mol as previously described [12]. Thiolated IL4R $\alpha$ monoclonal antibodies (BD Biosciences, San Diego, CA), which specifically binds to CD124 (also known as the $\alpha$ subunit of mouse IL4R $\alpha$ ), were conjugated to maleimide-functionalized SPIONs as previously described [13]. The antiSPION-IL4R $\alpha$ were purified on magnetic-activated cell sorting columns (Miltenyi Biotec, Bergisch Gladbach, Germany). The hydrodynamic size and zeta potential (i.e., surface charge) of nanoparticles before and after their conjugation with IL4R $\alpha$ antibodies were measured using Zetasizer Nano ZS90 (Malvern Instruments, Malvern, UK). The amount of IL4R $\alpha$ antibodies conjugated to SPION was quantified as described elsewhere [13].

\section{Tumor cell culture}

$4 \mathrm{~T} 1$ murine breast cancer cells (CRL-2539, American Type Culture Collection [ATCC], Manassas, VA) were cultured in complete Roswell Park Memorial Institute 1,640 medium containing 10\% gamma-irradiated fetal bovine serum and 100 units / mL penicillin-streptomycin (Life Technologies, Carlsbad, CA) at $37^{\circ} \mathrm{C}$ in a humidified atmosphere containing $5 \% \mathrm{CO}_{2}$. These cancer cells were chosen since their tumor growth in vivo and metastatic spread in mice closely mimics stage IV human breast cancer [14]. SPION-IL4R $\alpha$ and DOX were applied at a concentration of $5 \mu \mathrm{g} / \mathrm{mL}$ each based on our previous studies [13,15].

\section{Cell viability assessments}

Cell viability was analyzed by 3-(4,5-dimethylthiazol2-yl)-2,5-diphenyltetrasodium bromide (MTT) assay as per the manufacturer's protocol (Merck Millipore, Billerica, MA). Upon completion of incubation of cultured $4 \mathrm{~T} 1$ cells in complete medium with either anti-IL4R $\alpha$ antibodies, SPION-IgG1 (nanoparticles with isotype antibodies), SPION-IL4R $\alpha$, DOX, or combined SPION-IL4R $\alpha+D O X$ incubation for 24,48 , and 72 hours, $10 \mu \mathrm{L}$ of MTT reagent was added to each well and samples were incubated for 2 hours at $37^{\circ} \mathrm{C}$. The formazan crystals formed were dissolved in $100 \mu \mathrm{L}$ isopropanol with $0.04 \mathrm{~N} \mathrm{HCl}$ and the absorbance was measured within an hour using an Epoch BioTek ELISA plate reader (BioTek, Winoo- 
ski, VT) with an absorbance wavelength of $570 \mathrm{~nm}$ and a reference wavelength of $630 \mathrm{~nm}$. The percentage of cell viability under each condition was calculated relative to controls.

\section{Thiobarbituric acid reactive substances assay}

A thiobarbituric acid reactive substances (TBARS; R\&D Systems, Minneapolis, MN) parameter assay kit was used to measure the overall oxidative stress in $4 \mathrm{~T} 1$ cells caused by either SPION-IL4R $\alpha$, DOX, or combined SPION-IL4R $\alpha+D O X$ at different time intervals relative to controls. Briefly, cultured $4 \mathrm{~T} 1$ cells were equilibrated overnight, treated, washed and lysed using a cell lysis buffer. Cells were incubated with an acid reagent for 15 minutes and centrifuged, after which supernatants were treated with thiobarbituric acid reagent for 2-3 hours at $45-50^{\circ} \mathrm{C}$. The absorbance of the samples was measured at $532 \mathrm{~nm}$ using a BioTek enzyme-linked immunosorbent assay plate reader.

\section{Assessments of apoptosis}

The percentages of live, early apoptotic, late apoptotic, total apoptotic, and dead cells were quantitatively evaluated using Muse Annexin V and Dead Cell Assay kit (Merck Millipore) before and after treatment of 4T1 tumor cells with either SPION-IL4R $\alpha$, DOX, or combined SPION-IL4R $\alpha+$ DOX. These experiments were conducted since apoptosis/ programmed cell death is an important regulator of cell growth and proliferation. Cells were mixed with the reagent, incubated for 20 minutes and analyzed using a Muse Cell Analyzer (Merck Millipore).

\section{Specific targeting and interaction assessments}

To confirm specific targeting of SPION-IL4R $\alpha$ to $4 \mathrm{~T} 1$ cells, blocking of IL4R $\alpha$ receptors was performed for 4 hours with anti-mouse IL4R $\alpha$ antibody/isotype IgG1 antibody in the presence or absence of IL-4. The specific targeting of antiIL4R $\alpha$ conjugated SPION was assessed in vitro using cultured $4 \mathrm{~T} 1$ cells. Nanoparticles were labeled with fluorescein isothiocyanate (FITC) using a Pierce FITC labeling kit as per the manufacturer's protocols (Thermo Fisher Scientific, Waltham, MA). Cultured and trypsinized 4T1 cells were equilibrated overnight, fixed with $4 \%$ paraformaldehyde, washed with phosphate buffered saline (PBS) and blocked with antimouse IL4R $\alpha$ antibody or isotype IgG1 antibody, respectively, for 30 minutes at room temperature on a shaker. The cells were washed with ice-cold PBS and stained with FITC labeled SPION-IL4R $\alpha$ (SPION-IL4R $\alpha$-FITC) for 30 minutes at room temperature on a shaker. The wells were washed again three times with ice-cold PBS, and mounted using UltraCruz mounting medium (Santa Cruz Biotechnology
Inc., Dallas, TX) containing DAPI nuclear stain. All immunocytology slides were screened using an Olympus BX53 fluorescent microscope (Olympus Corporation, Tokyo, Japan). Fluorimetry measurements were performed in 96 well black tissue culture plates under the same conditions. Briefly, approximately $10^{3}$ cells/ well were equilibrated overnight, after which cells were blocked for 4 hours with either antiIL4R $\alpha$ antibodies or isotype IgG1 antibodies. Fluorescence assessments were performed on an Epoch BioTek plate reader to determine differences in fluorescence intensity.

In addition to the above, additional experiments were conducted to assess the viability and frequency of apoptosis following blocking of IL4R $\alpha$ receptors with anti-mouse IL4R $\alpha$ antibody or isotype IgG1 antibody, respectively, for 2 hours at room temperature on a shaker. The cells were then washed with PBS and the cell viability and apoptosis frequency was analyzed as described above after 12 hours of incubation using either anti-IL4R $\alpha$ antibodies, SPION-IgG1, SPIONIL4R $\alpha$, DOX, or combined SPION-IL4R $\alpha+$ DOX.

\section{Statistical analysis}

Data are presented as the mean values \pm standard error. All data were analyzed by $t$ tests using the SPSS ver. 12.0 software (SPSS Inc., Chicago, IL). A p $<0.05$ was considered significant for all tests. All experiments were performed in triplicate with exposure incubation times of 12, 24, 48, and 72 hours as applicable and results were compared to untreated cells.

\section{Results}

\section{SPION-IL4R $\alpha$ design and characterization}

PEG-functionalized SPIONs were conjugated to anti-IL4R $\alpha$ antibodies via a PEG chain with a molecular weight of 2,000 $\mathrm{g} / \mathrm{mol}$. SPIONs have a hydrodynamic size of $132.5 \pm 5.9 \mathrm{~nm}$ and a surface charge of $2.71 \pm 0.62 \mathrm{mV}$ based on measurement of the zeta potential using a Zetasizer Nano ZS90. Following conjugation with anti-IL4R $\alpha$ antibodies, no significant change in the size of SPION-IL4R $\alpha$ nanocarriers (i.e., $133.1 \pm 5.7$ $\mathrm{nm}$ ) was measured, although their surface charge decreased slightly to $1.05 \pm 0.46 \mathrm{mV}$. Quantification by bicinchoninic acid protein assay revealed that the amount of conjugated IL4R $\alpha$ antibodies was approximately $13.7 \mu \mathrm{g}$ per milligram of SPION. Further details regarding characterization of these nanoparticles have been published elsewhere [13]. 
A
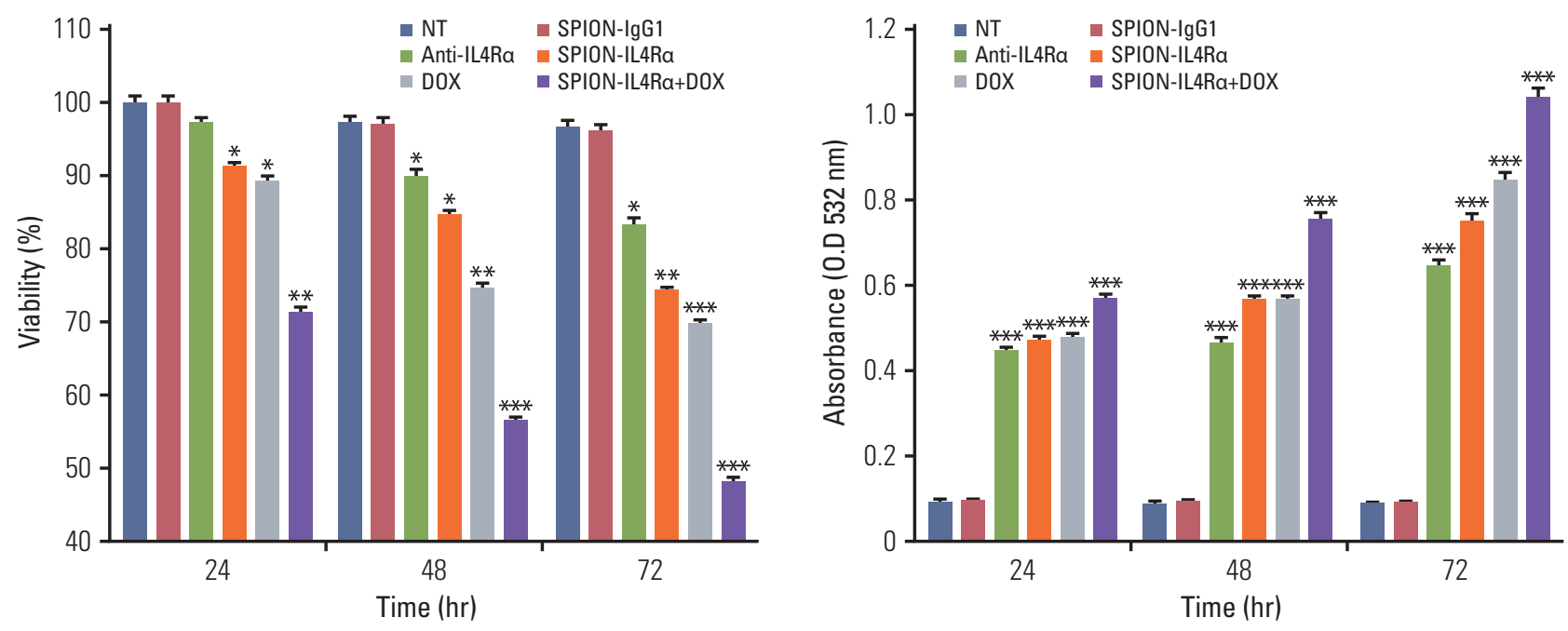

Fig. 1. Cell viability and oxidative stress generation assessments of the in vitro effects of SPION-IL4R $\alpha$, DOX, or combined SPION-IL4R $\alpha+$ DOX. MTT assay (A) and TBARS assay (B) were performed to assess cell viability and oxidative stress generation, respectively, in $4 \mathrm{~T} 1$ cancer cells. Analyses were performed after incubation for 24, 48, and 72 hours. Data are expressed as the mean \pm standard error, ${ }^{*} \mathrm{p}<0.05,{ }^{* *} \mathrm{p}<0.01$, ${ }^{* * *} \mathrm{p}<0.001$. NT, no treatment; SPION-IL4R $\alpha$, superparamagnetic iron oxide nanoparticles conjugated with anti-interleukin-4 receptor $\alpha$ [IL4R $\alpha$ ] blocking antibodies; DOX, doxorubicin; MTT, 3-(4,5-dimethylthiazol-2-yl)-2,5-diphenyltetrasodium bromide; TBARS, thiobarbituric acid reactive substances.

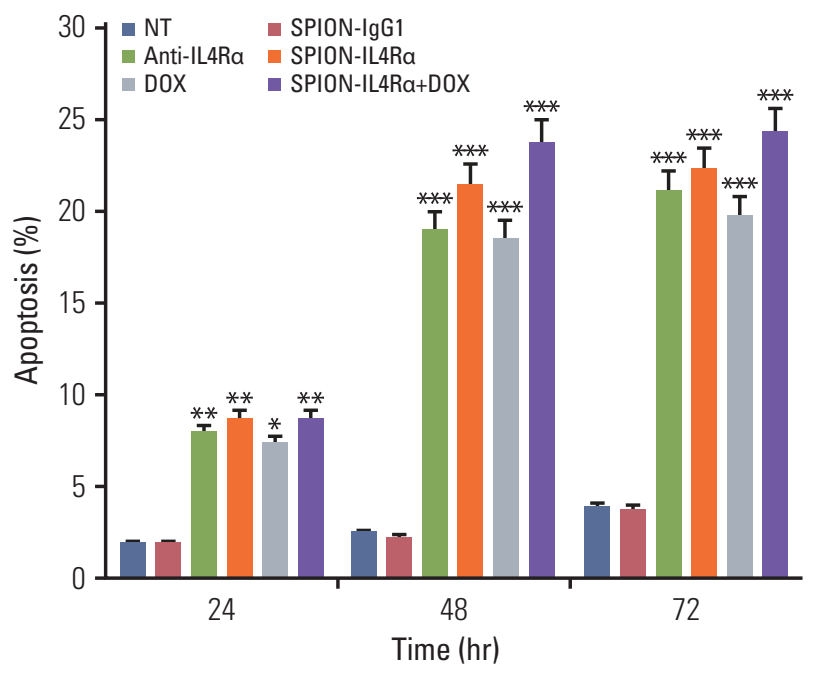

Fig. 2. Assessments of apoptosis caused by SPION-IL4Ra, DOX, and SPION-IL4R $\alpha+$ DOX. Analyses were performed after incubation for 24,48 , and 72 hours. Data are expressed as the mean \pm standard error, ${ }^{*} \mathrm{p}<0.05,{ }^{* *} \mathrm{p}$ $<0.01,{ }^{* * *} \mathrm{p}<0.001$. NT, no treatment; SPION-IL4R $\alpha$, superparamagnetic iron oxide nanoparticles conjugated with anti-interleukin-4 receptor $\alpha$ [IL4R $\alpha$ ] blocking antibodies; DOX, doxorubicin.

\section{Combination of SPION-IL4R $\alpha$ and DOX induces cyto- toxicity and increases reactive oxygen species in $4 \mathrm{~T} 1$ cancer cells}

Next, the ability of SPION-IL4R $\alpha$ to induce tumor cell death and apoptosis was assessed and compared to the effect of DOX or SPION-IL4R $\alpha+$ DOX. SPION-IgG1 nanocarriers and anti-IL4R $\alpha$ antibodies alone were also used. MTT cell proliferation assays and TBARS assessments were conducted to determine if SPION-IL4R $\alpha$ and / or DOX can induce cytotoxicity and oxidative stress in cultured 4T1 cancer cells, respectively. A significant reduction in cell viability was observed after cell incubation with either SPION-IL4R $\alpha$ or DOX separately, while a more prominent effect was observed in response to combined treatment (Fig. 1). Following 72-hour incubation of SPION-IL4R $\alpha+$ DOX, cell viability decreased to $48 \%$. Similarly, while SPION-IL4R $\alpha$ and DOX caused comparable elevations in reactive oxygen species when administered alone, substantial increases in oxidative stress were observed in response to their combined administration. 

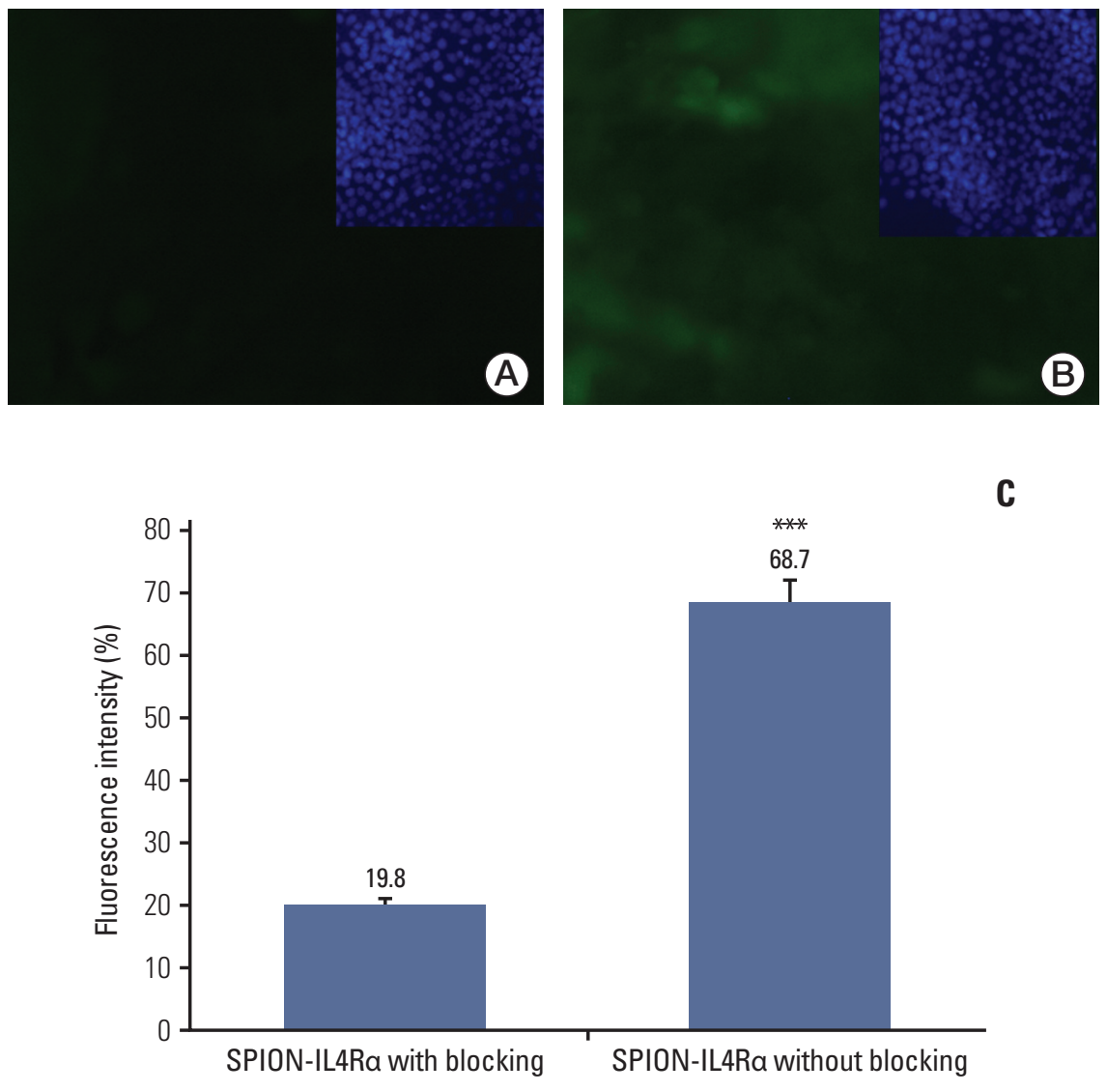

Fig. 3. In vitro interaction studies of SPION-IL4R $\alpha$ with IL4R $\alpha$ receptors on $4 \mathrm{~T} 1$ cells. Fluorescence intensity decreased upon blocking of receptors with anti-IL4R $\alpha$ antibodies (A) while higher fluorescence intensity was observed upon blocking using isotype IgG antibody (B). (C) Fluorimetry data showing the difference in fluorescence intensity after blocking with either the anti-IL4R $\alpha$ antibody or isotype IgG antibody (i.e., without blocking). All immunofluorescence images were captured at the same fluorescence exposure time of 300 milliseconds. Blocking was performed with anti-IL4R $\alpha$ antibodies. FITC-conjugated SPION-IL4R $\alpha$ were used to show the expression and appear in green; blue staining indicates staining of nuclei with DAPI. Data are expressed as the mean \pm standard error, ${ }^{* * *} \mathrm{p}<0.001$. SPION-IL4R $\alpha$, superparamagnetic iron oxide nanoparticles conjugated with anti-interleukin-4 receptor $\alpha$ [IL4R $\alpha]$ blocking antibodies; FITC, fluorescein isothiocyanate.

\section{Combination of SPION-IL4R $\alpha$ and DOX increased apop- tosis frequency in $4 \mathrm{~T} 1$ cancer cells}

The apoptosis frequency induced by SPION-IgG1, antiIL4R $\alpha$ antibodies, SPION-IL4R $\alpha$, and DOX in breast cancer murine 4T1 cells was also evaluated. To determine if apoptosis induced by SPION-IL4R $\alpha$ is potentiated by the addition of DOX, the effects of SPION-IL4R $\alpha+\mathrm{DOX}$ were investigated. While anti-IL4R $\alpha$ antibodies, SPION-IL4R $\alpha$, DOX, and SPION-IL4R $\alpha+$ DOX caused a significant increase in cell death at 24 hours, as expected, no significant increases in apoptosis were observed in response to SPION-IgG1. SPION-IL4R $\alpha$ and DOX alone caused significant increases in programmed cell death from 48 hours onwards, while
SPION-IL4R $\alpha+$ DOX induced more apoptosis than either compound alone (Fig. 2). The higher levels of apoptosis induced by SPION-IL4R $\alpha+$ DOX after 24 hours indicated an additive effect of this combination compared to either treatment alone.

\section{SPION-IL4R $\alpha$ successfully interacted with IL4R $\alpha$ recep- tors on $4 \mathrm{~T} 1$ cancer cells}

To confirm whether the synthesized SPION-IL4R $\alpha$ indeed bound to IL4R $\alpha$ receptors on $4 \mathrm{~T} 1$ cells, cultured cells were blocked with anti-IL4R $\alpha$ /isotype IgG1 antibody for 4 hours prior to exposure to SPION-IL4R $\alpha$-FITC conjugated nanocarriers. Variations in fluorescence intensity were assessed 
microscopically and through fluorimetry assay. Prior blocking of the IL4R $\alpha$ receptors with anti-IL4R $\alpha$ antibodies inhibited binding of SPION-IL4R $\alpha$-FITC and consequently caused a decrease in fluorescence intensity (Fig. 3). In 4T1 cells, which have been treated with isotype IgG1 antibody, a higher fluorescence intensity was found. This result was as expected since the IL $4 \mathrm{R} \alpha$ receptors were not blocked. This increased fluorescence intensity was also confirmed and quantified by fluorimetry assessments, which revealed a $68.7 \%$ fluorescence intensity without blocking, while the fluorescence intensity was estimated to be $19.8 \%$ following IL4R $\alpha$ blocking, confirming that the developed nanocarriers positively bound to IL4R $\alpha$ receptors on cultured $4 \mathrm{~T} 1$ cells.

Interestingly, upon prior blocking of cells with anti-IL4R $\alpha$ antibodies, an increase in cell viability and decrease in apoptosis was observed in $4 \mathrm{~T} 1$ cells treated with SPION-IL4R $\alpha$ nanocarriers, providing additional confirmation of the specific targeting of the designed nanocarriers. These findings also indicate that prior blocking with anti-IL4R $\alpha$ antibodies blocked the IL $4 \mathrm{R} \alpha$ receptors, leaving no/minimal sites for the nanocarriers to bind. In contrast, 4T1 cells that were blocked with isotype IgG1 antibodies showed significantly decreased cell viability and increased apoptosis (Fig. 4A and B). Since DOX acts independently of SPION-IL4R $\alpha$ nanocarriers, it caused a significant decrease $(\mathrm{p}<0.05)$ in cell viability and increase in apoptosis (as expected), regardless of whether the receptors were blocked with either anti-IL4R $\alpha$ antibodies or isotype IgG1 antibodies. Overall, these results conclusively confirmed that our developed nanoparticles did indeed bind to IL4R $\alpha$ receptors on cultured $4 \mathrm{~T} 1$ cells.

\section{Discussion}

The interaction of IL4R $\alpha$ receptor with the cytokine IL- 4 has been shown to be associated with proliferation of breast cancer cells [16,17], causing enhanced survival and metastases of cancer cells in vivo [18]. IL-13, a cytokine related to IL-4, also uses IL4R $\alpha$ receptors for cell signaling. IL4R $\alpha$ receptors have been found to be expressed and activated in mouse models of breast cancer [19]. The possibility that both IL-4 and IL-13 can be simultaneously targeted in breast cancer is based on the idea that they both play a role in cell proliferation and are dependent on the IL4R $\alpha$ chain, either for recognition or signal transduction. Therefore, disruption of the interaction of IL- 4 with IL4R $\alpha$ receptors may help abrogate the proliferation and metastatic spread of cancer cells.

In the present study, we assessed the effects of blocking IL4R $\alpha$ receptors in $4 \mathrm{~T} 1$ breast cancer cells by specifically and actively targeting an antibody-conjugated SPION nanocar-

A
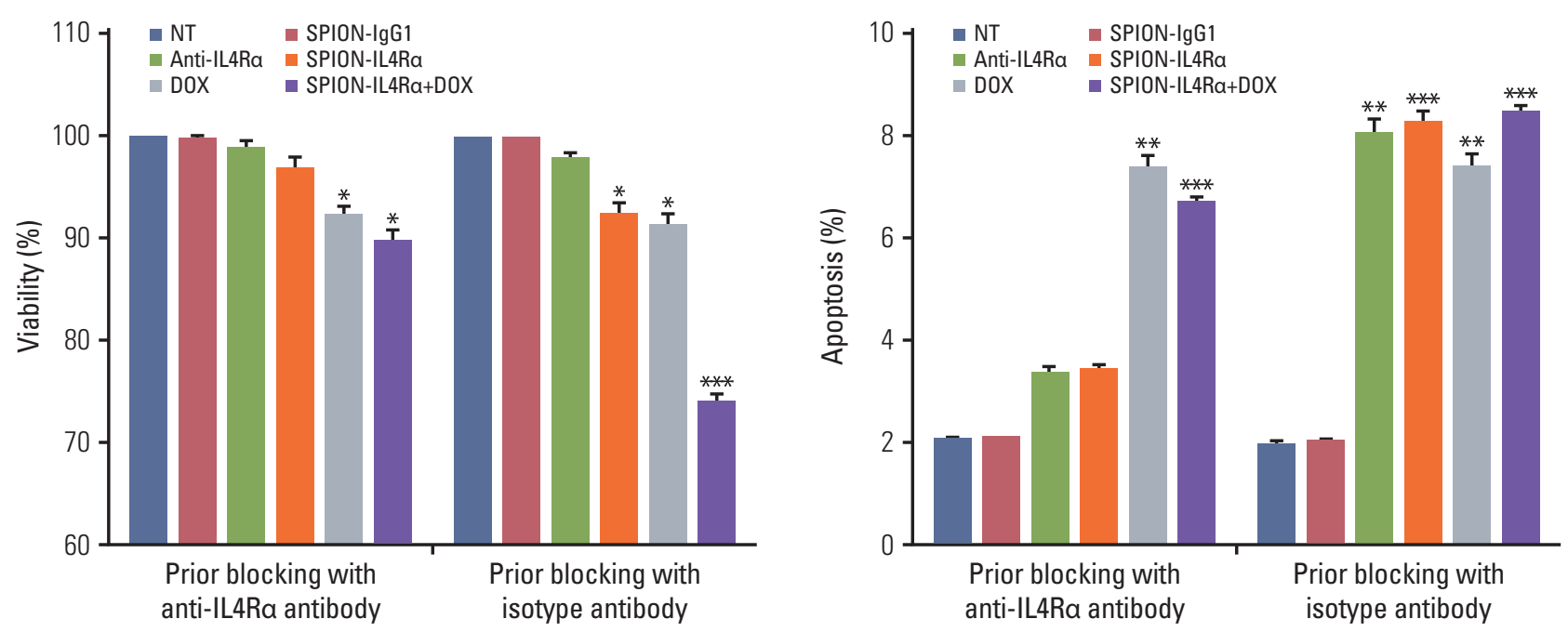

Fig. 4. Assessments of cell viability (A) and apoptosis (B) following prior blocking of IL4R $\alpha$ receptors on 4 T1 cells. Blocking was performed for 1 hour with either anti-IL4R $\alpha$ antibody or isotype IgG antibody (i.e., without blocking) and assessments were performed after 12 hours of incubation following treatment with SPION-IgG1, anti-IL4R $\alpha$ antibodies alone, SPIONIL4R $\alpha$, DOX, and SPION-IL4R $\alpha+D O X$. Data are expressed as the mean \pm standard error, ${ }^{*} \mathrm{p}<0.05,{ }^{* *} \mathrm{p}<0.01,{ }^{* * *} \mathrm{p}<0.001$. NT, no treatment; SPION-IL4R $\alpha$, superparamagnetic iron oxide nanoparticles conjugated with anti-interleukin-4 receptor $\alpha$ [IL4R $\alpha$ ] blocking antibodies; DOX, doxorubicin. 
rier and compared these effects with combined treatment with DOX, a chemotherapeutic drug widely used in cancer therapy. With this novel approach, we aim to significantly improve both cancer diagnosis and therapy while minimizing the side effects of currently used chemotherapeutic agents. SPIONs offer several advantages for prospective in vivo applications over competing nanocarriers by enabling both single cell tracking and imaging of diseased areas using noninvasive MRI. Additionally, SPIONs have superior biocompatibility and ability to cross biological membranes in addition to a high drug-loading capacity and enabling sustained release, enhanced drug stability and absorption, as well as targeted deposition.

The nanocarriers developed here have been previously validated to be highly safe and revealed excellent stability at neutral $\mathrm{pH}$, while their stability decreased remarkably in acidic environments with the use of 2,000 g/mol PEG chain linker; thus, they can preferentially release conjugated blocking antibodies [13].

This is the first study to evaluate the ability of SPIONIL4R $\alpha$ alone or in combination with DOX to induce cytotoxicity and increase reactive oxygen species generation and apoptosis frequency in breast cancer cells. One of the major hindrances to successful cancer treatment is the resistance of cancer cells to cell death, which primarily occurs because of drug resistance mechanisms, enhanced efflux of anti-cancer drugs or alterations in cell signaling-pathways enabling cancer cells to quickly undergo metastasis [18]. Therefore, induction of apoptosis plays a major role in limiting the spread of cancer cells [20]. Since IL-4 plays a role in survival of cancer cells, we hypothesized that neutralization of the interaction of IL4R $\alpha$ receptors through the use of IL4R $\alpha$ antibodies conjugated to SPION may limit cell proliferation and induce apoptosis in cancer cells. Moreover, the main anticancer action of DOX is thought to be a result of topoisomerase II inhibition and free radical generation [21].

In agreement with other studies [19,22], the current study demonstrated that blockade of IL4R $\alpha$ successfully induced apoptosis of $4 \mathrm{~T} 1$ cancer cells. Similarly and as expected, DOX also induced apoptosis of $4 \mathrm{~T} 1$ cells, confirming the results of other studies [23,24]. It is important to note that potentiation of apoptosis was observed when a combination of SPIONIL4R $\alpha$ and DOX was used. This additive effect of IL-4-neutralizing antibody in SPION-IL4R $\alpha$ when combined with chemotherapy may enable considerable reduction of tumor growth via increased apoptotic events, thus limiting metastasis and minimizing the required amount of chemotherapeutic drugs.

Advances in novel DDSs to diagnose, target and treat local and metastatic tumor sites have provided many advantages in limiting tumor progression and metastases. In this study, the in vitro efficacy of a combination of SPION-IL4R $\alpha$ and
DOX on $4 \mathrm{~T} 1$ breast cancer cells that multiply and simulate stage IV human breast cancer was evaluated. SPION-IL4R $\alpha$ was used because $4 \mathrm{~T} 1$ cancer cells have been shown to overexpress IL4R $\alpha$ receptors, which in turn use IL- 4 and IL-13 to mediate signal transduction events that modulate cell proliferation [19]. Therefore, simultaneous inhibition of the interaction of IL-4 and IL-13 by blocking IL4R $\alpha$ receptors using anti-IL4R $\alpha$ antibody nanocarriers may offer a promising therapeutic approach in breast cancer. A nanocarrier-based approach was used to enable improvement of treatment efficacy owing to specific targeting of cells while minimizing side effects. The specific targeting of designed SPION-IL4R $\alpha$ nanocarriers was demonstrated through a combination of immunofluorescence, fluorimetry, cell viability, and apoptosis assessments following prior blocking of IL4R $\alpha$ receptors on $4 \mathrm{~T} 1$ cells with either anti-IL4R $\alpha$ antibody or isotype IgG antibody (i.e., without blocking). Additionally, a commonly used anti-cancer drug, DOX, was used to investigate if the combination of SPION-IL4R $\alpha$ and DOX was able to achieve a higher treatment efficacy than either treatment alone.

\section{Conclusion}

The current study conclusively confirmed that a combination of SPION-IL4R $\alpha$ and DOX induced better cytotoxicity, oxidative stress and apoptosis in breast cancer cells than either treatment alone. Both SPION-IL4R $\alpha$ and DOX caused alterations in the levels of inflammatory mediators produced by $4 \mathrm{~T} 1$ cancer cells. In addition, the synthesized SPIONIL4R $\alpha$ successfully targeted the IL4R $\alpha$ receptors on 4T1 cancer cells. These positive study results are encouraging, and future studies will now focus on the in vivo efficacy of SPION-IL4R $\alpha$ in the breast cancer mouse model.

\section{Conflicts of Interest}

Conflict of interest relevant to this article was not reported.

\section{Acknowledgments}

The authors would like to extend their sincere appreciation to the Deanship of Scientific Research at King Saud University for funding this Research (Group No. RG1435-013). 


\section{References}

1. DeSantis CE, Lin CC, Mariotto AB, Siegel RL, Stein KD, Kramer JL, et al. Cancer treatment and survivorship statistics, 2014. CA Cancer J Clin. 2014;64:252-71.

2. Ben-Baruch A. Host microenvironment in breast cancer development: inflammatory cells, cytokines and chemokines in breast cancer progression: reciprocal tumormicroenvironment interactions. Breast Cancer Res. 2003;5: 31-6.

3. DeNardo DG, Barreto JB, Andreu P, Vasquez L, Tawfik D, Kolhatkar N, et al. CD4(+) T cells regulate pulmonary metastasis of mammary carcinomas by enhancing protumor properties of macrophages. Cancer Cell. 2009;16:91102.

4. Hosoyama T, Aslam MI, Abraham J, Prajapati SI, Nishijo $\mathrm{K}$, Michalek JE, et al. IL-4R drives dedifferentiation, mitogenesis, and metastasis in rhabdomyosarcoma. Clin Cancer Res. 2011;17:2757-66.

5. Joshi BH, Leland P, Lababidi S, Varrichio F, Puri RK. Interleukin-4 receptor alpha overexpression in human bladder cancer correlates with the pathological grade and stage of the disease. Cancer Med. 2014;3:1615-28.

6. Joshi BH, Leland P, Asher A, Prayson RA, Varricchio F, Puri RK. In situ expression of interleukin-4 (IL-4) receptors in human brain tumors and cytotoxicity of a recombinant IL-4 cytotoxin in primary glioblastoma cell cultures. Cancer Res. 2001;61:8058-61.

7. Al Faraj A, Shaik AS, Al Sayed B, Halwani R, Al Jammaz I. Specific targeting and noninvasive imaging of breast cancer stem cells using single-walled carbon nanotubes as novel multimodality nanoprobes. Nanomedicine (Lond). 2016;11: 31-46.

8. Davis ME, Chen ZG, Shin DM. Nanoparticle therapeutics: an emerging treatment modality for cancer. Nat Rev Drug Discov. 2008;7:771-82.

9. Rosen JE, Chan L, Shieh DB, Gu FX. Iron oxide nanoparticles for targeted cancer imaging and diagnostics. Nanomedicine. 2012;8:275-90.

10. Tiwari G, Tiwari R, Sriwastawa B, Bhati L, Pandey S, Pandey $\mathrm{P}$, et al. Drug delivery systems: an updated review. Int J Pharm Investig. 2012;2:2-11.

11. Tacar O, Sriamornsak P, Dass CR. Doxorubicin: an update on anticancer molecular action, toxicity and novel drug delivery systems. J Pharm Pharmacol. 2013;65:157-70.

12. Al Faraj A, Shaik AP, Shaik AS. Effect of surface coating on the biocompatibility and in vivo MRI detection of iron oxide nanoparticles after intrapulmonary administration. Nanotoxicology. 2015;9:825-34.

13. Al Faraj A, Shaik AS, Afzal S, Al-Muhsen S, Halwani R. Specific targeting and noninvasive magnetic resonance imaging of an asthma biomarker in the lung using polyethylene glycol functionalized magnetic nanocarriers. Contrast Media Mol Imaging. 2016;11:172-83.

14. Yang S, Zhang JJ, Huang XY. Mouse models for tumor metastasis. Methods Mol Biol. 2012;928:221-8.

15. Al Faraj A, Shaik AP, Shaik AS. Magnetic single-walled carbon nanotubes as efficient drug delivery nanocarriers in breast cancer murine model: noninvasive monitoring using diffusion-weighted magnetic resonance imaging as sensitive imaging biomarker. Int J Nanomedicine. 2015;10:157-68.

16. Roca H, Craig MJ, Ying C, Varsos ZS, Czarnieski P, Alva AS, et al. IL-4 induces proliferation in prostate cancer PC3 cells under nutrient-depletion stress through the activation of the JNK-pathway and survivin up-regulation. J Cell Biochem. 2012;113:1569-80.

17. Zhang WJ, Li BH, Yang XZ, Li PD, Yuan Q, Liu XH, et al. IL-4-induced Stat6 activities affect apoptosis and gene expression in breast cancer cells. Cytokine. 2008;42:39-47.

18. Todaro M, Lombardo Y, Francipane MG, Alea MP, Cammareri $\mathrm{P}$, Iovino $\mathrm{F}$, et al. Apoptosis resistance in epithelial tumors is mediated by tumor-cell-derived interleukin-4. Cell Death Differ. 2008;15:762-72.

19. Venmar KT, Carter KJ, Hwang DG, Dozier EA, Fingleton B. IL4 receptor ILR4alpha regulates metastatic colonization by mammary tumors through multiple signaling pathways. Cancer Res. 2014;74:4329-40.

20. Hengartner MO. The biochemistry of apoptosis. Nature. 2000; 407:770-6.

21. Hurley LH. DNA and its associated processes as targets for cancer therapy. Nat Rev Cancer. 2002;2:188-200.

22. Roth F, De La Fuente AC, Vella JL, Zoso A, Inverardi L, Serafini P. Aptamer-mediated blockade of IL4Ralpha triggers apoptosis of MDSCs and limits tumor progression. Cancer Res. 2012;72:1373-83.

23. Niu G, Zhu L, Ho DN, Zhang F, Gao H, Quan Q, et al. Longitudinal bioluminescence imaging of the dynamics of doxorubicin induced apoptosis. Theranostics. 2013;3:190-200.

24. Bao L, Haque A, Jackson K, Hazari S, Moroz K, Jetly R, et al. Increased expression of $\mathrm{P}$-glycoprotein is associated with doxorubicin chemoresistance in the metastatic 4T1 breast cancer model. Am J Pathol. 2011;178:838-52. 